\title{
Symbol Insertion: A Low-Complexity Joint Peak Power Reduction and Forward Error Correction for Single-Carrier Systems
}

\author{
Makoto Tanahashi, Student Member, IEEE, and Hideki Ochiai, Member, IEEE
}

\begin{abstract}
In this paper, we present a new modulation code, named symbol insertion (SI), that simultaneously achieves both peak-to-average power ratio (PAPR) reduction and forward error correction for pulse-shaped single-carrier systems in a low-complexity manner. Based on the fact that PAPR highly depends on the successive phase patterns of discrete complex symbols, the proposed code controls phase shifts by inserting a redundant symbol between consecutive information-bearing symbols. At the receiver, by viewing the inserted symbols as parities, we exploit them for error correction. We show that, when the SI is used as an inner code of serially concatenated codes, its error rate performance is close to those of conventional capacity-approaching codes. The proposed system thus becomes favorable if the amount of PAPR reduction is taken into account. Furthermore, the SI with a rotationally invariant insertion scheme can accommodate itself to noncoherent detection.
\end{abstract}

Index Terms-Peak-to-average power ratio reduction, turbo decoding, noncoherent communications, rotational invariance

\section{INTRODUCTION}

$\mathbf{S}$ INGLE-carrier phase shift keying (PSK) modulation is an attractive air interface with relatively low peak-to-average power ratio (PAPR). However, since the dynamic range of the modulated signals significantly affects power amplifier efficiency, PAPR reduction schemes play an important role even in the PSK framework.

Although the PSK symbols have a unit amplitude, the envelope of the band-limited signals fluctuates as a result of pulse-shaping filtering. It is known that the resulting PAPR highly depends on the successive phase patterns of underlying discrete complex symbols [1], and thus it can be reduced by the use of a signal constellation with small maximum phase transition. By limiting the maximum phase transition to 135 degrees, $\pi / 4$-shift QPSK can achieve a PAPR reduction of approximately $0.5 \mathrm{~dB}$ with a moderate roll-off factor of pulseshaping filter. However, such an approach is less effective for high-order PSK constellations. Another interesting approach is the use of Ternary-PSK (TPSK or 3-PSK) [2]. The TPSK

Paper approved by ... Manuscript received ... ; revised ...

This paper was in part presented at the IEEE International Conference on Communications 2008 (ICC'08), Beijing, China, May 2008 and at the International Symposium on Information Theory and its Applications 2008 (ISITA'08), Auckland, New Zealand, December 2008. This work was in part supported by the Japan Society for the Promotion of Science (JSPS) Research Fellowships for Young Scientists and MEXT KAKENHI 21760279.

The authors are with the Department of Electrical and Computer Engineering, Yokohama National University, 79-5 Tokiwadai, Hodogaya, Yokohama, Kanagawa 240-8501, Japan (e-mail: makoto@ochiailab.dnj.ynu.ac.jp; hideki@ynu.ac.jp)

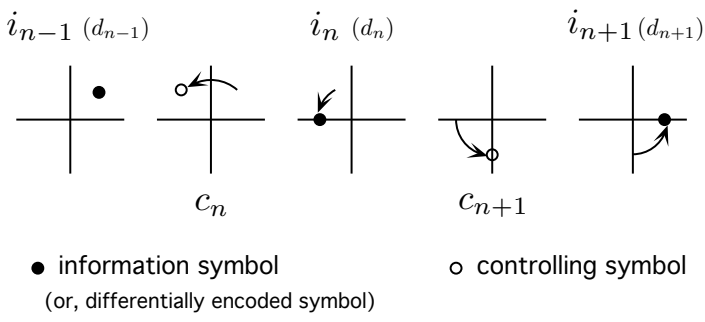

Fig. 1. The proposed symbol insertion system. Large phase rotation can be eliminated by the presence of judiciously inserted controlling symbols.

constellation consists of the three signal points with equilateral triangular geometry. The maximum phase transition is thus restricted to $360 / 3=120$ degrees, and the resulting PAPR is lower than that of $\pi / 4$-shift QPSK. The concept of TPSK can be more generally extended to regular $M$-sided polygons where $M$ is an odd number. However, as $M$ increases, this approach becomes less effective in terms of PAPR reduction capability, similar to those with constellation rotation.

Since these constellation-oriented techniques have limited peak power control capability, several sequence-oriented approaches based on modulation codes [3-5] have been proposed recently. In $[3,4]$, effective constraining patterns of phases for reducing PAPR are studied, but their systematic implementation of encoders and decoders appears to be challenging. The trellis shaping approach (see [5] and references therein) can effectively reduce PAPR, but at the cost of high complexity at the transmitter.

In this paper, as a low-complexity alternative to the existing sequence-oriented techniques, we propose a new system called symbol insertion (SI) illustrated in Fig. 1. The SI controls phase shifts (and thus reduces the associated PAPR) by inserting a (redundant) controlling symbol between consecutive information-bearing symbols, where the controlling symbol to be inserted is determined and tabulated beforehand for a given pair of anteroposterior symbols. The encoding complexity of the SI is therefore almost negligible. Due to the insertion of controlling symbols, the maximum allowable phase transition can be reduced to as small as $180 / 2=90$ degrees in any signal constellation. Hence, the resulting PAPR is lower than those of the above mentioned constellation-oriented schemes.

At first glance, the loss of data rate associated with the insertion of redundant symbols appears prohibitive, considering the fact that it requires twice as large bandwidth as those without an SI constraint for achieving the same data rate. This also 
indicates that it requires twice as large signal-to-noise ratio (SNR) per information bit to achieve a certain error rate. From an information-theoretic perspective, constraining symbols is equivalent to embedding memory, and it is well recognized that such an input to a channel in turn reduces channel capacity (or more precisely, average mutual information (AMI)), from those without constraint. Nevertheless, this drawback can be compensated for by the SI itself from the following viewpoint: the inserted symbols can be viewed as parities for informationbearing symbols, which thus allows us to exploit them for error correction at the receiver. In a block diagram notation, the SI can be represented as a convolutional code (CC) having a trellis structure as sketched in Fig. 2 (a). The major difference of this CC from widely adopted CCs is that it operates with an $M$-ary alphabet, instead of binary, for an $M$-ary constellation. Due to this trellis structure, the SI can be efficiently decoded by the Viterbi or BCJR [6] algorithms.

The decoding complexity is low since the SI is a CC with only one delay element. This fact, however, also implies that this code does not necessarily have a good code structure for error correction. Nevertheless, if the system is combined with another $\mathrm{CC}$ as illustrated in Fig. 3, it can then be viewed as an instance of serially concatenated convolutional codes [7], and thus iterative decoding is applicable.

In a serially concatenated convolutional code, if an inner code has a recursive (i.e., feed-back) structure, it can enjoy socalled interleaver gain. This is demonstrated in, e.g., [8] with a simple differential encoder (DE) as an inner code. Motivated by this fact, we consider the feed-back type encoder depicted in Fig. 2 (b), where the insertion operation is performed on differentially encoded symbols. This encoding structure also allows us to employ a noncoherent iterative decoder similar to the work in [9-12]. In what follows, we refer to the straightforward realization of SI shown in Fig. 2 (a) as feedforward symbol insertion (FFSI) and the recursive version with the DE shown in Fig. 2 (b) as feed-back symbol insertion (FBSI).

This paper is organized as follows: after introduction of the overall system model in Section II, an AMI expression of this system is derived in Section III. This can serve as a theoretical limit for achievable performance of the SI. Section IV is devoted to a description of soft-input soft-output decoding of the SI, which is necessary to achieve the error performance close to the predicted AMI limit. In Section V, we present design criteria to identify good insertion tables in terms of PAPR reduction capability and error rate performance. A specific design example for 8 -PSK and its simulation results are given in Section VI. Finally, Section VII concludes the paper.

For simplicity, we restrict our attention to an $M$-PSK system over additive white Gaussian noise (AWGN) channel with either coherent or noncoherent detection. Nevertheless, it should be noted that any constellation format can be combined with the SI, and it is also applicable to fading channels by appropriately modifying the decoding metrics derived in this paper.
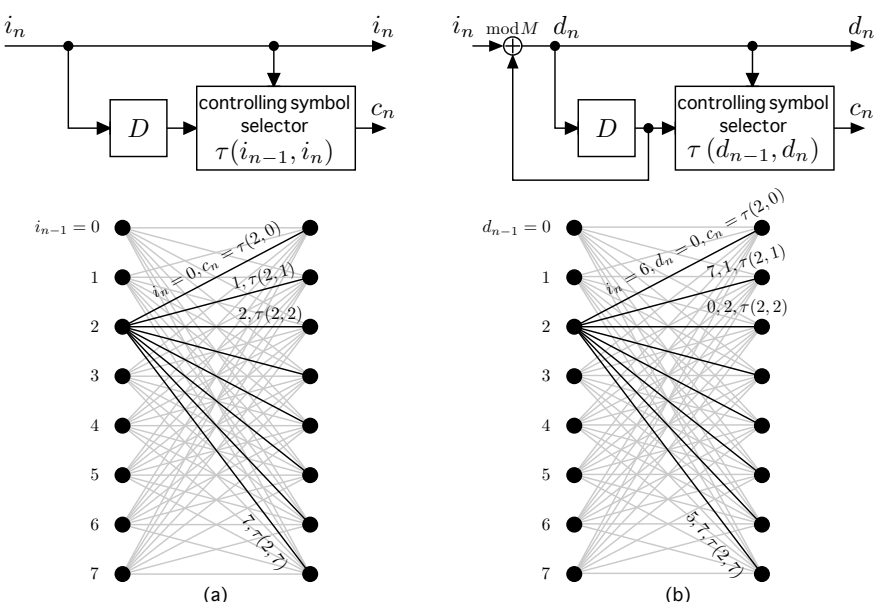

Fig. 2. Block and trellis diagrams (in the case of $M=8$ ) of symbol insertion systems. (a) Feed-forward symbol insertion (FFSI). (b) Feed-back symbol insertion (FBSI).

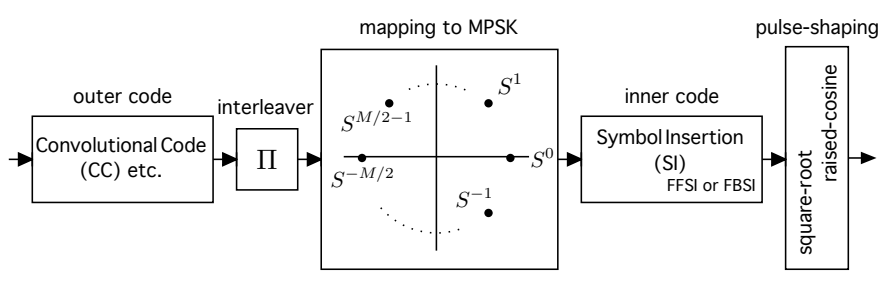

Fig. 3. A transmitter model with $M$-PSK modulation.

\section{SySTEM MODEL}

The transmitter model considered throughout this paper is depicted in Fig. 3. In this transmitter, source data is encoded by a channel code (typically, a convolutional code), and mapped onto $M$-PSK constellation after random interleaving. A Gray labeling is assumed for constellation mapping as an example. The SI works as an inner component code in this serially concatenated code and carries out its encoding operation for the $M$-PSK symbols after constellation mapping. A bandlimited baseband signal is generated by passing the output symbols through a pulse-shaping filter.

\section{A. Transmit Signal Generation}

Let us denote an $M$-PSK constellation by

$$
\mathcal{S}_{M}=\left\{S^{i}=\sqrt{E_{s}} e^{j 2 \pi i / M}, i \in \mathbb{Z}_{M}\right\},
$$

where an integer variable $i$ denotes the constellation index and $E_{s}$ is the energy of one transmit symbol. The energy of one transmit bit is denoted by $E_{b}$. Let $\boldsymbol{i}=\left\{i_{n} \in \mathbb{Z}_{M}, 1 \leq n \leq\right.$ $N\}$ be a sequence of the indices of consecutive symbols of length $N$, representing information after channel coding and interleaving. We consider the two insertion operation schemes shown in Fig. 2: (a) $\boldsymbol{i}$ is fed directly to the channel (FFSI), and (b) $\boldsymbol{i}$ is differentially encoded into $\boldsymbol{d}=\left\{d_{n} \in \mathbb{Z}_{M}, 0 \leq\right.$ $n \leq N\}$ (FBSI) as

$$
d_{n}=d_{n-1} \oplus i_{n}
$$


where $d_{0} \triangleq 0$ is a non-data carrying symbol and the operator $\oplus$ denotes modulo- $M$ addition. Likewise, modulo- $M$ subtraction will be denoted by $\ominus$. For a given information sequence $\boldsymbol{i}$ or its differentially-encoded counterpart $\boldsymbol{d}$, the controlling symbol selector in Fig. 2 determines a controlling symbol index $c_{n}$ to be inserted between $i_{n-1}$ and $i_{n}$ (or $d_{n-1}$ and $\left.d_{n}\right)$. In the following, we explicitly denote this function by $\tau(\cdot, \cdot)$ so that the insertion symbol index is given by

$$
c_{n}=\tau\left(i_{n-1}, i_{n}\right),
$$

for the FFSI case. The final form of the transmitted symbol sequence $S$ is given by

$$
\begin{aligned}
\boldsymbol{S} & =\left\{S^{c_{1}}, S^{i_{1}}, \ldots, S^{c_{n}}, S^{i_{n}}, \ldots, S^{c_{N}}, S^{i_{N}}\right\} \\
& =\left\{\boldsymbol{S}_{1}, \ldots, \boldsymbol{S}_{n}, \ldots, \boldsymbol{S}_{N}\right\},
\end{aligned}
$$

where $\boldsymbol{S}_{n} \triangleq\left\{S^{c_{n}}, S^{i_{n}}\right\}$. Note that $i_{n}$ is replaced by $d_{n}$ in the case of the FBSI.

For a given impulse response of the pulse-shaping filter, $g(t)$, and a symbol duration $T_{s}$, the band-limited (i.e., continuous-time) transmit signal $s(t)$ is expressed as

$$
s(t)=\sum_{n=1}^{2 N} A_{n} g\left(t-(n-1) T_{s}\right),
$$

where we have defined $A_{n}$ as $A_{n}=S^{c_{(n+1) / 2}}$ for $n$ odd and $A_{n}=S^{i_{n} / 2}$ for $n$ even. We assume the widely-used square-root raised-cosine (RRC) pulse shape for $g(t)$. The SI can reduce the envelope fluctuation of $s(t)$ and the associated PAPR without changing the bandwidth of $s(t)$ (aside from the simple doubling due to transmitting twice as many symbols), since we do not change the parameters of the band-limiting filter $g(t)$.

\section{B. Channel Model for Error Rate Performance Evaluation}

Assuming matched filtering and perfect symbol timing synchronization at the receiver, we consider a discrete-time AWGN channel model for error rate performance evaluation. Specifically, let $\boldsymbol{W}$ denote a noise sequence with the two-sided power spectral density $N_{0} / 2$. The initial carrier phase at the receiver, denoted by $\theta$, is assumed constant over the entire length of $\boldsymbol{S}$. A received symbol sequence $\boldsymbol{R}$ is then expressed as

$$
\boldsymbol{R}=\boldsymbol{S} e^{j \theta}+\boldsymbol{W}
$$

We denote a pair of the received symbols at the $n$th time slot by $\boldsymbol{R}_{n} \triangleq\left\{R_{n}^{(c)}, R_{n}^{(i)}\right\}$, which corresponds to the received version of $\boldsymbol{S}_{n}=\left\{S^{c_{n}}, S^{i_{n}}\right\}$.

In this paper, we consider the following three scenarios depending on the knowledge of the carrier phase $\theta$ at the receiver:

1) $\theta$ is known at the receiver, and thus always can be forced to 0 without loss of generality (coherent case).

2) $\theta$ is partially known, i.e., limited to integer multiples of $2 \pi / M$. This is a model of $M$-ary phase ambiguity caused by the false lock of phase locked-loop (PLL). Since the phase ambiguity is eliminated by the use of the
DE at the transmitter, we call this model differentially coherent case.

3) There is no phase acquisition assumed, i.e., $\theta$ is unknown and uniformly distributed over $[0,2 \pi$ ) (noncoherent case).

Due to the DE structure and rotationally invariant property of the insertion function $\tau(\cdot, \cdot)$ which will be introduced later in Section V-A, the FBSI has an advantage that it can work with both the differentially coherent and noncoherent cases.

\section{AVERAge Mutual Information AnALYsis}

Since the SI embeds memory into the input to a channel, achievable information rate (i.e., AMI) should be always lower than those without any constraint. This section provides an efficient numerical method to compute the AMI subject to the SI constraint of a given insertion function $\tau(\cdot, \cdot)$. Some example AMI values will be numerically evaluated in Section VI-D. In this section, only the FFSI encoder model is considered in our subsequent analysis for simplicity, but we note that the presence of the DE does not change the resulting AMI.

\section{A. Preliminaries}

In general, the AMI between $S$ and $\boldsymbol{R}$ is calculated by

$$
I(\boldsymbol{S} ; \boldsymbol{R})=H(\boldsymbol{R})-H(\boldsymbol{R} \mid \boldsymbol{S})
$$

where $H(\boldsymbol{X})$ denotes the (differential) entropy of a stochastic process $\boldsymbol{X}$. The first term denoting the entropy of the received symbols is, by definition, expressed as

$$
H(\boldsymbol{R})=-\lim _{n \rightarrow \infty} \frac{1}{2 n} E\left[\log _{2} p\left(\boldsymbol{R}_{1}^{n}\right)\right],
$$

where we have introduced the notation $\boldsymbol{R}_{1}^{n} \triangleq\left(\boldsymbol{R}_{1}, \cdots, \boldsymbol{R}_{n}\right)$ for convenience, and $p(\cdot)$ is the joint probability density function (pdf) associated with its argument. In general, the evaluation of (8) becomes prohibitively complex as $n$ increases. However, for transmitted symbols with a specific stochastic structure, a novel computational technique developed in [13] can be used for the calculation of (8). In the case of the SI with an $M$-ary alphabet, we carry out the following procedure.

Let us rewrite $p\left(\boldsymbol{R}_{1}^{n}\right)$ as a joint pdf of $\boldsymbol{R}_{1}^{n}$ with a state of the SI at the $n$th time instant, where the state is defined as a stored symbol in the delay element of Fig. 2. Hence,

$$
p\left(\boldsymbol{R}_{1}^{n}\right)=\sum_{j=0}^{M-1} p\left(\boldsymbol{R}_{1}^{n}, i_{n}=j\right) .
$$


Then, $\alpha_{n}(j) \triangleq p\left(\boldsymbol{R}_{1}^{n}, i_{n}=j\right)$ can be decomposed as

$$
\begin{aligned}
\alpha_{n}(j)= & \sum_{k=0}^{M-1} p\left(i_{n}=j, \boldsymbol{R}_{n} \mid i_{n-1}=k, \boldsymbol{R}_{1}^{n-1}\right) \cdot \alpha_{n-1}(k) \\
= & \sum_{k=0}^{M-1} p\left(\boldsymbol{R}_{n} \mid i_{n}=j, i_{n-1}=k, \boldsymbol{R}_{1}^{n-1}\right) \\
& \cdot p\left(i_{n}=j \mid i_{n-1}=k, \boldsymbol{R}_{1}^{n-1}\right) \cdot \alpha_{n-1}(k) \\
= & \frac{1}{M} \sum_{k=0}^{M-1} p\left(\boldsymbol{R}_{n} \mid i_{n}=j, i_{n-1}=k, \boldsymbol{R}_{1}^{n-1}\right) \cdot \alpha_{n-1}(k) \\
= & \frac{1}{M} \sum_{k=0}^{M-1} \frac{p\left(\boldsymbol{R}_{1}^{n} \mid i_{n}=j, i_{n-1}=k\right)}{p\left(\boldsymbol{R}_{1}^{n-1} \mid i_{n}=j, i_{n-1}=k\right)} \cdot \alpha_{n-1}(k) \\
=\frac{1}{M} \sum_{k=0}^{M-1} \frac{p\left(R_{n-1}^{(i)}, R_{n}^{(c)}, R_{n}^{(i)} \mid i_{n-1}=k, c_{n}=\tau(k, j), i_{n}=j\right)}{p\left(R_{n-1}^{(i)} \mid i_{n-1}=k\right)} & \cdot \alpha_{n-1}(k) .
\end{aligned}
$$

\section{B. Coherent Case}

Let $p_{\theta}(R \mid S)$ be the channel transition pdf of the AWGN channel with the carrier phase $\theta$ taken into account. It is then given by

$$
p_{\theta}(R \mid S)=\frac{1}{\pi N_{0}} \exp \left(-\frac{\left|R-S e^{j \theta}\right|^{2}}{N_{0}}\right) .
$$

With this, in the case of the coherent detection with $\theta=0$, $\alpha_{n}(j)$ can be reduced to

$$
\alpha_{n}(j)=\frac{1}{M} \sum_{k=0}^{M-1} p_{0}\left(R_{n}^{(i)} \mid S_{i_{n}}^{j}\right) p_{0}\left(R_{n}^{(c)} \mid S_{c_{n}}^{\tau(k, j)}\right) \cdot \alpha_{n-1}(k),
$$

where the notations $S_{i_{n}}^{j}$ and $S_{c_{n}}^{\tau(k, j)}$ are the shorthands for the events $S^{i_{n}}=S^{j}$ and $S^{c_{n}}=S^{\tau(k, j)}$, respectively. The second term of (7), denoting the entropy of a white Gaussian noise with variance $N_{0} / 2$, is given by the closed form:

$$
H(\boldsymbol{R} \mid \boldsymbol{S})=H(\boldsymbol{W})=\log _{2}\left(\pi e N_{0}\right) .
$$

Using (7) to (13), one can compute an AMI- $E_{s} / N_{0}$ curve for a given insertion function $\tau(\cdot, \cdot)$. The calculated AMI$E_{s} / N_{0}$ characteristic is also valid for the FBSI over the differentially coherent channel.

\section{Noncoherent Case}

In this subsection, extending the discussions given in [14, 15] to our SI case, we derive the AMI in the case of the noncoherent detection. It is well known that noncoherent channel capacity (or AMI) is augmented by increasing the length of observed symbols for detection, at the price of exponential growth in decoding complexity $[14,15]$. Thus, we derive AMI for a given observed symbol duration $L$. Note that $L$ is defined such that it includes the number of the controlling symbols. On the other hand, we define $l$ as the observed information (or differentially-encoded) symbols. These two lengths are related as

$$
L=2 l+1, \quad l=(L-1) / 2 .
$$

Since in our SI framework every information symbol is associated with a single trellis section, the required number of the trellis states is given by $M^{l}$. In the conventional schemes for iterative decoding of noncoherently detected multiple symbols (often called turbo DPSK), the required complexity for a given observed length is much larger than that of the SI. For example, in the framework of [10-12], $L$ consecutive symbols are simultaneously observed with $M^{L-1}$-state trellis. The significant reduction in the number of the required states stems obviously from the presence of the deterministically inserted symbols. Hence, the SI can increase the effective observation length without any additional expansion of the trellis size.

In the case of $l=1$ (and hence $L=3$ ), we obtain the $\alpha$-value (10) as

$$
\begin{aligned}
\alpha_{n}(j)= & \frac{1}{M} \sum_{k=0}^{M-1} \frac{\overline{p_{\theta}}\left(R_{n-1}^{(i)}, R_{n}^{(c)}, R_{n}^{(i)} \mid S_{i_{n-1}}^{k}, S_{c_{n}}^{\tau(k, j)}, S_{i_{n}}^{j}\right)}{\bar{p}_{\theta}\left(R_{n-1}^{(i)} \mid S_{i_{n-1}}^{k}\right)} \\
\cdot \alpha_{n-1}(k), &
\end{aligned}
$$

where $\overline{p_{\theta}}\left(R_{1}, \cdots, R_{L} \mid S_{1}, \cdots, S_{L}\right)$ is a pdf with respect to $L$ consecutive symbols in which the unknown carrier phase $\theta$ is eliminated by averaging it over $[0,2 \pi)$. Hence,

$$
\begin{aligned}
\overline{p_{\theta}}\left(R_{1}, \cdots, R_{L} \mid S_{1}, \cdots, S_{L}\right) \triangleq & \frac{1}{2 \pi} \int_{0}^{2 \pi} \prod_{n=1}^{L} p_{\theta}\left(R_{n} \mid S_{n}, \theta\right) d \theta \\
= & \frac{1}{\left(\pi N_{0}\right)^{L}} \exp \left\{-\frac{1}{N_{0}} \sum_{n=1}^{L}\left(\left|R_{n}\right|^{2}+\left|S_{n}\right|^{2}\right)\right\} \\
& \cdot I_{0}\left(\frac{2}{N_{0}}\left|\sum_{n=1}^{L} R_{n} S_{n}^{*}\right|\right)
\end{aligned}
$$

where $I_{0}(\cdot)$ is a zeroth-order modified Bessel function of the first kind. The $\alpha$-value with respect to an arbitrary observation interval can be obtained by rederiving (15) with generalization of the state variable. Specifically, let us append the preceding $(l-1)$ states $i_{n-l+1}, \cdots, i_{n-1}$ to the current state $i_{n}$ and redefine a current state as $\boldsymbol{i}_{n}^{(l)} \triangleq\left(i_{n-l+1}, \cdots, i_{n}\right)$. The case with $l=1$ (and hence $L=3$ ) results in (15), and the case with $l=2$ (and hence $L=5$ ) leads to (17) at the top of the next page, where $\boldsymbol{j}=\left(j_{0}, j_{1}\right)$ is a state transitioning from the state $\boldsymbol{k}=\left(k, j_{0}\right)$. The expressions for $l>2$ can be derived in a straightforward manner. The derivation of the conditional entropy $H(\boldsymbol{R} \mid \boldsymbol{S})$ for the noncoherent case is given in the Appendix.

\section{Soft-Input Soft-Output Decoding}

The iterative decoding process of the proposed serial concatenation (Fig. 2) is the same as that given in [7], except that the SI has now an $M$-ary alphabet and thus mutual conversion between binary and $M$-ary messages is necessary. In this section, we describe soft-input soft-output (SISO) decoding of the SI.

\section{A. Mutual Conversion between Binary and M-ary Messages}

At the initial stage of each SISO decoding, a priori probabilities of $M$-ary constellation symbols denoted by $P_{a}\left(S^{i}\right), 0 \leq$ 


$$
\begin{aligned}
\alpha_{n}(\boldsymbol{j}) & \triangleq p\left(\boldsymbol{R}_{1}^{n}, \boldsymbol{i}_{n}^{(2)}=\boldsymbol{j}\right) \\
& =\frac{1}{M} \sum_{k=0}^{M-1} \frac{\overline{p_{\theta}}\left(R_{n-2}^{(i)}, R_{n-1}^{(c)}, R_{n-1}^{(i)}, R_{n}^{(c)}, R_{n}^{(i)} \mid S_{i_{n-2}}^{k}, S_{c_{n-1}}^{\tau\left(k, j_{0}\right)}, S_{i_{n-1}}^{j_{0}}, S_{c_{n}}^{\tau\left(j_{0}, j_{1}\right)}, S_{i_{n}}^{j_{1}}\right)}{\overline{p_{\theta}}\left(R_{n-2}^{(i)}, R_{n-1}^{(c)}, R_{n-1}^{(i)} \mid S_{i_{n-2}}^{k}, S_{c_{n-1}}^{\tau\left(k, j_{0}\right)}, S_{i_{n-1}}^{j_{0}}\right)} \cdot \alpha_{n-1}(\boldsymbol{k}) .
\end{aligned}
$$

$i<M$, are constructed from binary a priori probabilities $P_{a}\left(b^{j, i}\right), 0 \leq j<\log _{2} M$ as

$$
P_{a}\left(S^{i}\right)=\prod_{j=0}^{\log _{2} M-1} P_{a}\left(b^{j, i}\right),
$$

where the binary sequence $\left(b^{0, i}, b^{1, i}, \cdots, b^{\log _{2} M-1, i}\right)$ constitutes an $M$-ary symbol $S^{i}$.

Conversely, after the completion of each SISO decoding, binary a posteriori probabilities $P_{d}\left(b^{j}\right)$ are retrieved from $M$ ary a posteriori probabilities $P_{d}\left(S^{i}\right)$ as

$$
P_{d}\left(b^{j}\right)=\sum_{S \in \mathcal{S}^{j}} P_{d}(S)
$$

where $\mathcal{S}^{j}$ denotes a set of the symbols with their $j$ th constituent bit given by $b^{j}$.

\section{B. Branch Metric}

The main body of the SISO decoding is the BCJR algorithm that works on the SI trellis. Here, we describe branch metric definitions to run the BCJR algorithm.

Let $\gamma_{n}^{k \rightarrow j}$ denote the metric of the $j$ th branch diverging from the state $k$ at the $n$th trellis section. Basically, this is a quantity proportional to conditional pdfs $p\left(i_{n}=j, \boldsymbol{R}_{n} \mid i_{n-1}=\right.$ $\left.k, \boldsymbol{R}_{1}^{n-1}\right)$ for the FFSI decoder and $p\left(i_{n}=j, \boldsymbol{R}_{n} \mid d_{n-1}=\right.$ $k, \boldsymbol{R}_{1}^{n-1}$ ) for the FBSI case. In fact, a similar quantity has already appeared in (10), but now the a priori probability of an information symbol needs to be taken into account. We summarize the branch metric definitions for the FFSI/FBSI in coherent/noncoherent channels.

1) Coherent and Differentially Coherent Cases: According to (12), the branch metric for the FFSI with the coherent detection can be defined as

\section{FFSI, coherence:}

$$
\gamma_{n}^{k \rightarrow j}=P_{a}\left(S^{j}\right) \exp \left(-\frac{\left|R_{n}^{(i)}-S^{j}\right|^{2}+\left|R_{n}^{(c)}-S^{\tau(k, j)}\right|^{2}}{N_{0}}\right) .
$$

Note that we have omitted the quantities independent of the transition $k \rightarrow j$.

Due to the rotationally invariant property which will be introduced in Section V-A, the same metric is equally applicable to the FBSI with the differentially coherent detection, but with modification of variable according to the presence of the differential encoding:

\section{FBSI, differential coherence:}

$\gamma_{n}^{k \rightarrow j}=P_{a}\left(S^{j}\right) \exp \left(-\frac{\left|R_{n}^{(d)}-S^{j \oplus k}\right|^{2}+\left|R_{n}^{(c)}-S^{\tau(k, j \oplus k)}\right|^{2}}{N_{0}}\right)$.
2) Noncoherent Case: Regarding the noncoherent decoding of the FBSI, as in [10-12], we formulate our decoding metric based on the carrier-phase-averaged channel pdf given in (16). The branch metric is then given by

FBSI, noncoherence $(L=3)$ :

$$
\begin{aligned}
& \gamma_{n}^{k \rightarrow j}=P_{a}\left(S^{j}\right) \\
& \cdot \frac{I_{0}\left(\frac{2}{N_{0}}\left|R_{n-1}^{(d)} S^{k}+R_{n}^{(c)} S^{\tau(k, j \oplus k) *}+R_{n}^{(d)} S^{(j \oplus k) *}\right|\right)}{I_{0}\left(\frac{2}{N_{0}}\left|R_{n-1}^{(d)} S^{k *}\right|\right)} .
\end{aligned}
$$

Note that (22) corresponds to the case with $l=1(L=3)$. The metric for $l=2(L=5)$ can be derived from (17) in a similar manner.

\section{Design of Insertion PATtern}

In this section, we propose a systematic design rule of the insertion function $\tau(\cdot, \cdot)$ that can simultaneously achieve low PAPR and good error performance. The constellation size $M$ is limited to $M=2^{m}$ where $m \geq 2$. For notational convenience, we will restrict the range of the constellation index $i$ from $-\frac{M}{2}$ to $\frac{M}{2}-1$ (in a modulo- $M$ sense), instead of from 0 to $M-1$.

\section{A. Basic Rule for Insertion}

First, we introduce the property of rotational invariance [16] to the function $\tau(\cdot, \cdot)$. To this end, we define $\tilde{\tau}(i)$ as

$$
\tilde{\tau}(i) \triangleq \tau(0, i),
$$

and assume that $\tau(i, j)$ satisfies

$$
\tau(i, j)=i \oplus \tilde{\tau}(j \ominus i) .
$$

The above property indicates that the phase rotation $\tilde{\tau}(\cdot)$ given by a controlling symbol depends only on the index difference of the two adjacent symbols and not their individual values. Hence, for an arbitrary phase rotation integer $k$, we also have

$$
\tau(i \oplus k, j \oplus k)=\tau(i, j) \oplus k .
$$

Due to the introduction of this rule, we need only specify the controlling symbols to be inserted between the symbol of index 0 and the next symbols with $M$ index patterns.

Not only does this rotational invariance property make the systematic design feasible, it is in fact essential for the FBSI with the noncoherent and differentially coherent cases. Specifically, since a symbol sequence generated by the FBSI with this property is perfectly identified only from adjacent phase rotations and not from their absolute phases, the receiver can estimate the sequence even in the noncoherent case. Furthermore, there is no performance degradation incurred from the absolute phase uncertainty in the case of the differential coherence. Specifically, let $x \triangleq \theta \frac{M}{2 \pi} \in \mathbb{Z}_{M}$ denote a rotation 
caused by the carrier phase ambiguity. The sequence of FBSI indices given this rotation is then expressed as $\left\{\ldots, d_{n-1} \oplus\right.$ $\left.x, \tau\left(d_{n-1}, d_{n}\right) \oplus x, d_{n} \oplus x, \ldots\right\}$. Due to the property of (25), this sequence is equal to $\left\{\ldots, d_{n-1} \oplus x, \tau\left(d_{n-1} \oplus x, d_{n} \oplus\right.\right.$ $\left.x), d_{n} \oplus x, \ldots\right\}$, which represents a valid symbol sequence simply with the initial value of the DE $d_{0}$ replaced by $x$.

Let us denote the set of all possible distinct entries for $\tilde{\tau}(i)$ by $\mathcal{T}_{i}$. Then, without any constraint on the choice of $\tilde{\tau}(i)$, we have $\mathcal{T}_{i}=\left\{-\frac{M}{2}, \ldots, \frac{M}{2}-1\right\}$ and the cardinality of this set is $\left|\mathcal{T}_{i}\right|=M$ for each $i$. The insertion table can be defined by a length- $M$ vector composed of $\tilde{\tau}(i)$, i.e.,

$$
\tilde{\tau} \triangleq\left[\begin{array}{lll}
\tilde{\tau}\left(-\frac{M}{2}\right) & \cdots & \tilde{\tau}\left(\frac{M}{2}-1\right)
\end{array}\right] .
$$

The set of the possible table patterns is denoted by $\mathbb{T}$ and has $|\mathbb{T}|=\left|\mathcal{T}_{i}\right|^{M}=M^{M}$ distinct entries. Our objective is to find a suitable table $\tilde{\tau}$ from $\mathbb{T}$ that achieves both low PAPR and good error-correcting capability. Since the number of the possible entries in $\mathbb{T}$ grows exponentially, an exhaustive computer search becomes prohibitive even for moderate values of $M$. Therefore, we alternatively target a subset of $\mathbb{T}$ that may include low PAPR candidates.

\section{B. Insertion Table Candidates with Low PAPR}

As mentioned in the Introduction, it is known that PAPR highly depends on the successive phase patterns of transmitted symbols. For example, a phase shift close to 180 degrees causes a noticeable fluctuation of signal envelope and therefore contributes to high PAPR. From this perspective, we impose a constraint on the choice of the insertion table such that the maximum phase shift is less than 90 degrees. Specifically, for a given information symbol index $i$, the index of the inserted symbol $c \triangleq \tilde{\tau}(i)$ should satisfy

$$
\left|\arg S^{c}\right|,\left|\arg \frac{S^{c}}{S^{i}}\right| \leq 90 \text { degrees. }
$$

This is equivalent to imposing the following rule.

Rule 1: Minimization of maximum phase transition (MMP)

$$
\begin{gathered}
-\frac{M}{4} \leq c<\frac{M}{4}, \\
-\frac{M}{4} \leq c \ominus i<\frac{M}{4} .
\end{gathered}
$$

Under this rule, the set of possible candidates for the insertion table $\mathbb{T}^{\mathrm{MMP}}$ and its element-wise version $\mathcal{T}_{i}^{\mathrm{MMP}}$ are expressed as follows. First, $\mathcal{T}_{0}^{\mathrm{MMP}}$ is given by

$$
\mathcal{T}_{0}^{\mathrm{MMP}}=\left\{-\frac{M}{4}, \ldots, 0, \ldots, \frac{M}{4}\right\} .
$$

Thus, $\left|\mathcal{T}_{0}^{\mathrm{MMP}}\right|=\frac{M}{2}+1$. For $0<i<\frac{M}{2}, \mathcal{T}_{i}^{\mathrm{MMP}}$ is given by

$$
\mathcal{T}_{i}^{\mathrm{MMP}}=\mathcal{T}_{i-1}^{\mathrm{MMP}} \backslash\left\{-\frac{M}{4}+i-1\right\} .
$$

Thus, $\left|\mathcal{T}_{i}^{\mathrm{MMP}}\right|=\frac{M}{2}-i+1$. By a circular symmetry property of PSK constellation, $\left|\mathcal{T}_{i}^{\mathrm{MMP}}\right|$ for $-\frac{M}{2}<i<0$ is given by $\left|\mathcal{T}_{-i}^{\mathrm{MMP}}\right|=\left|\mathcal{T}_{M-i}^{\mathrm{MMP}}\right|$. Finally, we have $\mathcal{T}_{-\frac{M}{2}}^{\mathrm{MMP}}=\left\{-\frac{M}{4}, \frac{M}{4}\right\}$, but due to the symmetry property we can exclude the entry $-\frac{M}{4}$ without loss of generality. Therefore, we set $\left|\mathcal{T}_{-\frac{M}{2}}^{\mathrm{MMP}}\right|=1$. Consequently, the number of the entries in $\mathbb{T}^{\mathrm{MMP}}$ is given by

$$
\left|\mathbb{T}^{\mathrm{MMP}}\right|=\left|\mathcal{T}_{-\frac{M}{2}}^{\mathrm{MMP}}\right| \cdots\left|\mathcal{T}_{0}^{\mathrm{MMP}}\right| \cdots\left|\mathcal{T}_{\frac{M}{2}-1}^{\mathrm{MMP}}\right|=\left(\frac{M}{2}+1\right) \prod_{i=1}^{M / 2} i^{2} .
$$

We further reduce the number of targets by observing that too small phase shift also results in a high peak power, since adjacent shaping pulses are to be superimposed constructively. From this perspective, we add the following rule.

Rule 2: Zero-phase shift elimination (ZPE)

$$
c \ominus i \neq 0 \text {. }
$$

Let us count the possible entries in $\mathbb{T}^{\mathrm{MMP}-\mathrm{ZPE}}$ which is a subset of $\mathbb{T}^{\mathrm{MMP}}$. Since the entries 0 and $i$ are not allowed for $\mathcal{T}_{i}^{\text {MMP-ZPE }}$, we have

$$
\mathcal{T}_{i}^{\mathrm{MMP}-\mathrm{ZPE}}= \begin{cases}\mathcal{T}_{i}^{\mathrm{MMP}} \backslash\{0, i\}, & \text { for }-\frac{M}{4} \leq i<\frac{M}{4}, \\ \mathcal{T}_{i}^{\mathrm{MMP}}, & \text { otherwise }\end{cases}
$$

Thus, analogous to (32), the number of the entries in $\mathbb{T}^{\mathrm{MMP}-\mathrm{ZPE}}$ is given by

$$
\begin{aligned}
\left|\mathbb{T}^{\mathrm{MMP}-\mathrm{ZPE}}\right| & =\left|\mathcal{T}_{-\frac{M}{2}}^{\mathrm{MMP}-\mathrm{ZPE}}\right| \cdots\left|\mathcal{T}_{0}^{\mathrm{MMP}-\mathrm{ZPE}}\right| \cdots\left|\mathcal{T}_{\frac{M}{2}-1}^{\mathrm{MMP}-\mathrm{ZPE}}\right| \\
& =\frac{M}{2} \prod_{i=1}^{M / 4}\left(\frac{M}{2}-i-1\right)^{2} i^{2} .
\end{aligned}
$$

\section{PAPR Minimization}

To quantify the PAPR reduction capability of a given insertion table, we give a formal definition of PAPR with respect to the continuous-time signal $s(t)$ in (5) as follows.

The normalized instantaneous power of $s(t)$ is given by

$$
p(t)=\frac{|s(t)|^{2}}{P_{a v}},
$$

where $P_{a v}$ is the average power, i.e., $P_{a v}=E\left[|s(t)|^{2}\right]$. For $p(t)$ defined in this manner, we numerically calculate the complementary cumulative distribution function (CCDF) defined as $\operatorname{CCDF}(p)=\operatorname{Pr}[p(t)>p][17,18]$. The PAPR in our definition is then given by the value of $p$ at $\operatorname{CCDF}(p)=\varepsilon$ where the probability $\varepsilon$ is a small value such as $10^{-4}$.

In order to compute the CCDF by simulation, $s(t)$ in (5) is sampled $N_{s}$ times over every symbol interval $T_{s}$. Also, the pulse shape $g(t)$ is truncated to some symbol interval. We denote this duration by $K_{s}$.

\section{Coding Gain Maximization}

There are several possible criteria to choose a good insertion pattern from channel coding perspective. One straightforward approach is to optimize distance distribution of the codewords generated by the SI encoders [19]. However, the distance distribution does not serve as an appropriate measure for iterative decoding of the focused serially concatenated SI and $\mathrm{CC}$ that operates near the limit dictated by AMI. Alternatively, exploiting the fact that the AMI depends on a particular choice of an insertion pattern, we can conjecture that the one with 
the maximum AMI for a given operating SNR should result in the best performance, similar to the performance analysis of modulation codes [20]. However, the existence of iterative decoding with an outer $\mathrm{CC}$ should also be considered, but the AMI itself may not necessarily capture this effect.

To deal with this issue, we base our criterion on an EXIT curve [21] of the SI's SISO decoder presented in Section IV. The EXIT curve displays the AMI between the input bits to the SI's encoder (including constellation mapping) and the softbit output generated by the SISO decoder, when a simulated $a$ priori input is provided and $E_{s} / N_{0}$ is fixed. The simulated $a$ priori input of bits is fed, through the conversion (18), to the SISO decoder, and a posteriori output of bits are constructed from that of symbols using (19). With the EXIT curve obtained in this manner, we quantify the error correcting capability of a given insertion table as the threshold value of $E_{s} / N_{0}$ at which the curve of the SI with the examined insertion table and that of the outer $\mathrm{CC}$ touch.

\section{Performance Example with 8-PSK}

In this section, we show a design example with 8-PSK $(M=8)$ and evaluate its performance in terms of both PAPR and BER along with performance verification by AMI. Throughout simulation, we use the following $\mathrm{CC}$ with a constraint length $K=3$ as the outer CC of the focused serially concatenated system:

$$
\boldsymbol{G}_{c}=\left[\begin{array}{ccc}
1 & 0 & \frac{1+D^{2}}{1+D+D^{2}} \\
0 & 1 & \frac{1+D}{1+D+D^{2}}
\end{array}\right] .
$$

Since the rate of this $\mathrm{CC}$ is $2 / 3$ and that of the $\mathrm{SI}$ is $1 / 2$, the overall rate of the simulated 8-PSK system is $2 / 3 \cdot \log _{2} 8 \cdot 1 / 2=$ $1 \mathrm{bit} / \mathrm{symbol}$. The roll-off factor of the square-root raisedcosine filter is set to either 0.4 as an example of moderate band-limitation or 0.1 as a strict case.

The parameters used to compute PAPR characteristic is as follows: the oversampling number $N_{s}$ is set to 8 , and the pulse duration $K_{s}$ is determined such that 99.9 percent of all the energy falls within the range of $\left(-\frac{K_{s}}{2} T_{s}, \frac{K_{s}}{2} T_{s}\right]$. The specific values for the roll-off factors of 0.1 and 0.4 under this condition are found to be $K_{s}=12$ and 6 , respectively. The probability $\varepsilon$ to obtain a PAPR value from a CCDF chart is set to $10^{-4}$ as an example.

\section{A. Choice of Insertion Tables}

Computing (35) with $M=8$ follows that the number of possible insertion tables is only 64 , so that it is feasible to examine the PAPRs and the EXIT curves of all the candidates. First, we calculated their PAPRs based on the CCDF of normalized instantaneous power in the procedure described in Section V-C. From the channel coding perspective, we also calculated their threshold $E_{s} / N_{0}$ values based on EXIT curves. (The used SI model is the FBSI). Examples of CCDF and EXIT charts will be shown later in the following two subsections. The optimum insertion tables for the SI with rolloff factors 0.4 and 0.1 that minimize the sum (in $\mathrm{dB}$ ) of a

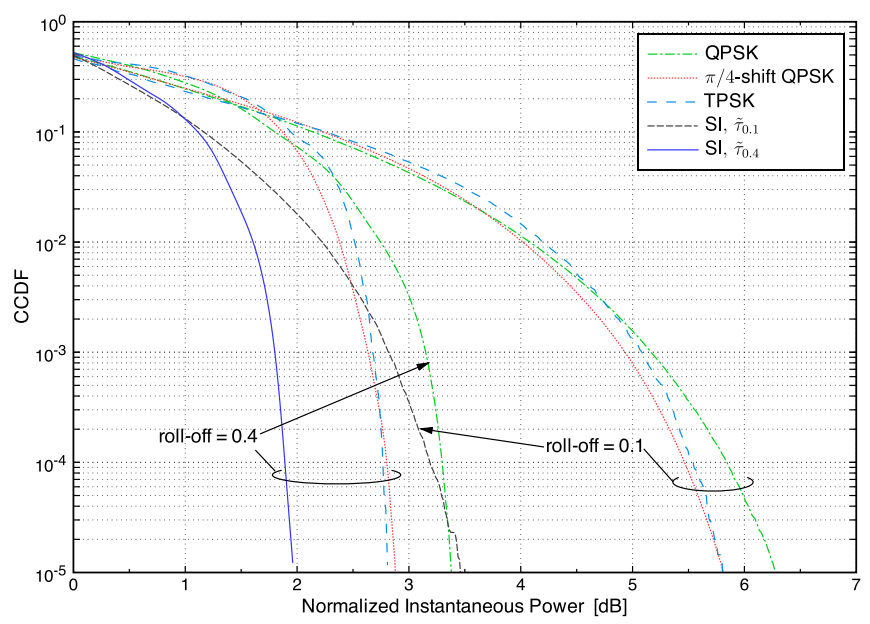

Fig. 4. CCDF of the normalized instantaneous power generated by 8-PSK with SI constraints.

PAPR and a threshold $E_{s} / N_{0}$ are listed in the following as $\tilde{\tau}_{0.4}$ and $\tilde{\tau}_{0.1}$, respectively:

$$
\begin{aligned}
\tilde{\boldsymbol{\tau}}_{0.4} & =\left[\begin{array}{llllllll}
2 & -2 & -1 & 1 & -2 & 2 & 1 & 1
\end{array}\right], \\
\tilde{\boldsymbol{\tau}}_{0.1} & =\left[\begin{array}{llllllll}
2 & -2 & -1 & 1 & 1 & -1 & 1 & 2
\end{array}\right] .
\end{aligned}
$$

Note that, as defined in (26), the entries of these insertion tables correspond (from left to right) to $\tilde{\tau}(-4), \tilde{\tau}(-3), \cdots, \tilde{\tau}(3)$.

We have observed that there is no significant difference in PAPR when the roll-off factor is 0.4 . Therefore, the table $\tilde{\tau}_{0.4}$, which in fact achieves the lowest threshold $E_{s} / N_{0}$, has been found to be the optimum one. On the other hand, the table $\tilde{\tau}_{0.1}$ achieves a smaller PAPR than the others in the case of the roll-off factor of 0.1 and also has a relatively low threshold $E_{s} / N_{0}$, thereby being the optimum insertion table for this rolloff factor setting.

\section{B. Peak-to-Average Power Ratio Reduction}

Figure 4 contains the plots of CCDF curves that show PAPR reduction capabilities of the chosen insertion tables. Since the SI poses almost no complexity at the transmitter, it may be reasonable to make comparison with conventional low-PAPR constellations such as $\pi / 4$-shift QPSK and TPSK [2] $]^{1}$.

The PAPR reduction (evaluated at $\mathrm{CCDF}=10^{-4}$ ) achieved by the SI over $\pi / 4$-shift QPSK is $0.9 \mathrm{~dB}$ for a roll-off factor of 0.4. On the other hand, for a roll-off of 0.1, the achievable PAPR reduction is more significant $(2.3 \mathrm{~dB})$. Therefore, it can be concluded that the proposed SI becomes more effective if the system requires higher bandwidth efficiency while only a limited signal processing capability for PAPR reduction is available at the transmitter.

\section{EXIT Chart}

In Fig. 5, the EXIT curves of the SI's SISO decoders operating with $\tilde{\boldsymbol{\tau}}_{0.4}$ and $\tilde{\boldsymbol{\tau}}_{0.1}$ are plotted along with that of the outer

\footnotetext{
${ }^{1}$ There are several more powerful PAPR reduction techniques available in the recent literature, e.g., [3-5]. These approaches, however, require higher complexity at the transmitter exclusively for PAPR reduction. Therefore, the comparison of the SI with these approaches is beyond the scope of the paper.
} 


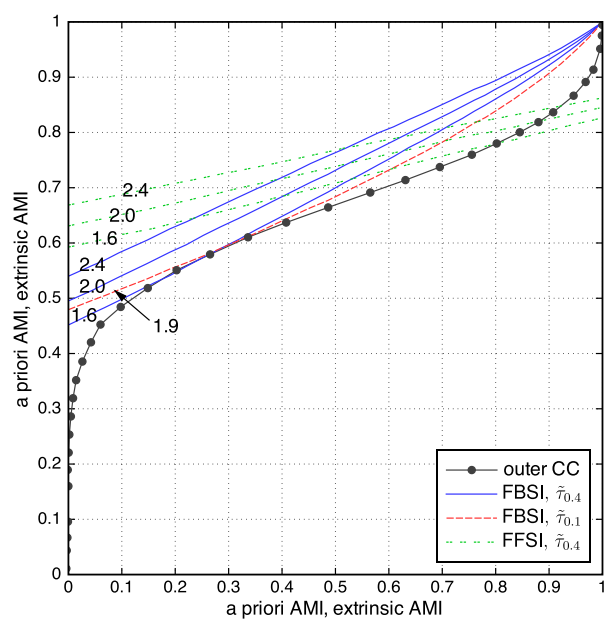

(a)

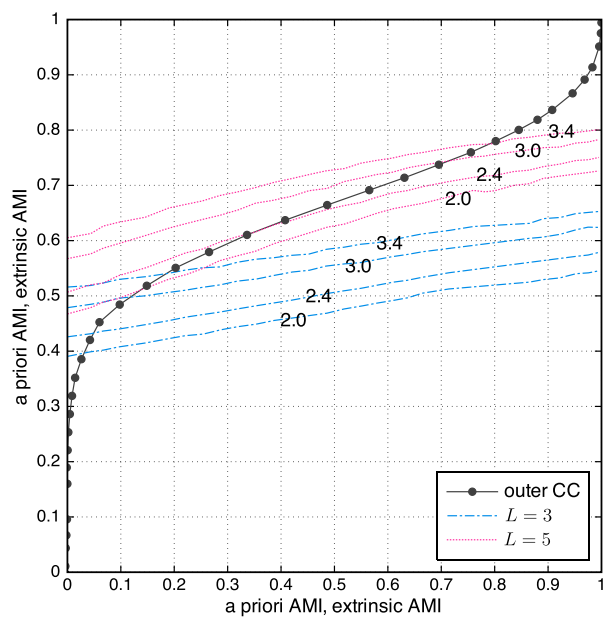

(b)

Fig. 5. EXIT chart of the SI's SISO decoder. The numbers attached to each trajectory represent operating $E_{s} / N_{0}$ (in $\mathrm{dB}$ ). (a) The FFSI for the coherent case and the FBSI for the differentially coherent case. (b) The FBSI for the noncoherent case where $\tilde{\tau}_{0.4}$ is used.

CC. It can be seen from these curves that iterative decoding converges only for the FBSI with the (differentially) coherent detection; the trajectories of the FFSI and even the FBSI, if the noncoherent detection is applied, have intersections with that of the outer CC. As will be seen later, this behavior results in the error rate performance with a noticeable error floor.

\section{Average Mutual Information}

To evaluate the AMI- $E_{s} / N_{0}$ characteristics subject to the SI constraints by $\tilde{\boldsymbol{\tau}}_{0.4}$ and $\tilde{\tau}_{0.1}$, we performed the numerical computation described in Section III. Figure 6 shows the results along with unconstrained 8-PSK and QPSK channel capacities. For the noncoherent case, the capacities of the unconstrained modulation are derived in [15].

From the results for the coherent case, we can remark that at low to moderate $E_{s} / N_{0}$, the degradation of $E_{s} / N_{0}$ inherent to the presence of the SI is less than $1 \mathrm{~dB}$. We have seen in Section VI-B that a PAPR reduction of more than $1 \mathrm{~dB}$

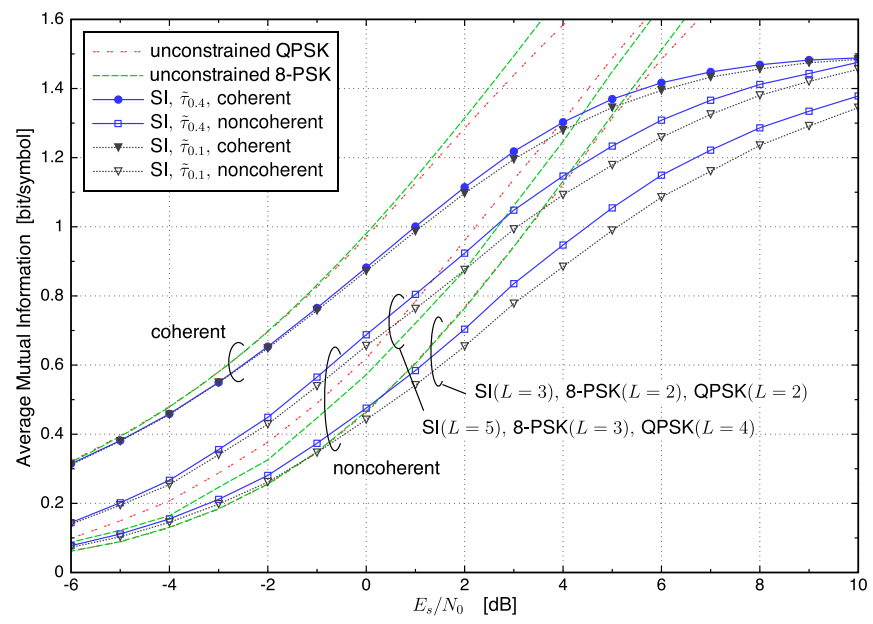

Fig. 6. AMI- $E_{s} / N_{0}$ characteristic of the SI with the insertion table $\tilde{\boldsymbol{\tau}}_{0.4}$ in the coherent/noncoherent cases.

is readily achieved as the roll-off factor of the pulse-shaping filter is decreased for higher bandwidth efficiency.

In the noncoherent case, we make a comparison under the constraint that the number of implied decoding trellis states is equal (or nearly equal). Recall that in the SI case, the decoding trellis has $M^{(L-1) / 2}$ states, and an unconstrained case has $M^{L-1}$ states (see Section III-C). The result shows that, unlike the coherent case, the SI in some information rate region outperforms the unconstrained systems, which can be inferred from the fact that the SI can extend the observation interval for a fixed decoding complexity. This result suggests that in addition to PAPR reduction, we can also reduce the required channel SNR for a certain transmission rate in the noncoherent case.

\section{E. Bit Error Rate in Coherent Transmission}

We examine the bit error rate (BER) performance of the serial concatenation of the SI and the outer $\mathrm{CC}$ in the coherent detection for the FFSI and the differentially coherent detection for the FBSI. In this simulation, the pseudo random interleaver is chosen from a class of S-random interleavers [22], the interleaver length is 9000 , the minimum separation value, $S$, is 12 , and the maximum count of iteration is set to 12 .

The simulation result is shown in Fig. 7, where we also plotted vertical lines representing the performance limits derived from the AMI- $E_{s} / N_{0}$ characteristics. As observed from the figure, the FFSI, which does not have a recursive structure, exhibits an error floor with no clear waterfall region. On the other hand, the FBSI, which has a recursive structure, achieves BER $=10^{-5}$ within $1.3 \mathrm{~dB}$ of the AMI limits with no error floor in the simulated range.

As a benchmark, we examine a conventional rate- $1 / 2$ (punctured) turbo code consisting of the following $K=4 \mathrm{CC}$ :

$$
\boldsymbol{G}_{c}=\left[\begin{array}{ll}
1 & \frac{1+D+D^{2}+D^{3}}{1+D^{2}+D^{3}}
\end{array}\right]
$$

with the same S-random interleaver (the same interleaver length and the value of $S$ ) as that employed in the proposed serial concatenation. In Fig. 7, we plot the BER of 


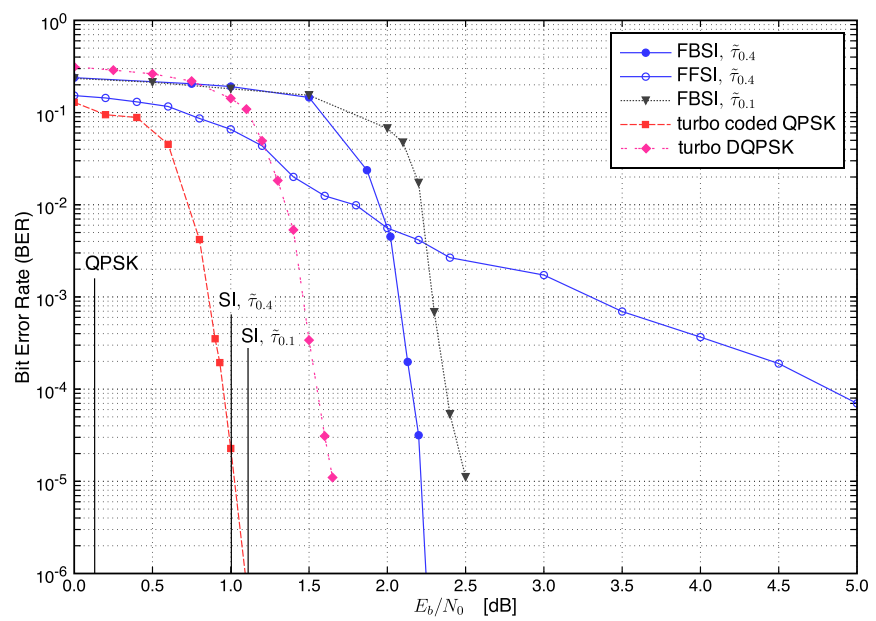

Fig. 7. BER of the FFSI in the coherent detection and the FBSI in the differentially coherent detection along with performance comparisons to the turbo coded QPSK and the turbo DQPSK.

this turbo code concatenated with ( $\pi / 4$-shift) QPSK in the coherent detection. This system has the same information rate $(1 \mathrm{bit} / \mathrm{symbol})$ as that of the proposed system. We also set the iteration count equal to that of the proposed serial concatenation. If we make a comparison between the FBSI with $\tilde{\tau}_{0.1}$ and this turbo coded QPSK, the former has a loss of $1.5 \mathrm{~dB}$ at $\mathrm{BER}=10^{-5}$ but it gains $2.3 \mathrm{~dB}$ from PAPR reduction, resulting in an overall gain of $0.8 \mathrm{~dB}$. In addition to this gain, it should be noted that the FBSI can operate in the differentially coherent detection whereas the conventional turbo code simulated here cannot.

To make a fair comparison in a differentially coherent scenario, we also examine turbo ( $\pi / 4$-shift) DQPSK [8], i.e., a serial concatenation of an outer $\mathrm{CC}$ and the DE as an inner code. The outer $\mathrm{CC}$ employed in this system is a rate- $1 / 2$ $K=3 \mathrm{CC}$ given by

$$
\boldsymbol{G}_{c}=\left[\begin{array}{ll}
1 & \frac{1+D^{2}}{1+D+D^{2}}
\end{array}\right] .
$$

Since the rate of the DE is 1 , the overall rate is given by $1 / 2 \cdot \log _{2} 4 \cdot 1=1 \mathrm{bit} / \mathrm{symbol}$, which is the same as that of the SI system under consideration. We also use the same S-random interleaver and iteration count as set in the other systems. Comparing the BERs of the FBSI and the turbo DQPSK reveals that the losses in $E_{b} / N_{0}$ at $\mathrm{BER}=10^{-5}$ is $0.6 \mathrm{~dB}$ and $0.9 \mathrm{~dB}$ for $\tilde{\boldsymbol{\tau}}_{0.4}$ and $\tilde{\boldsymbol{\tau}}_{0.1}$, respectively. These insignificant losses can be well compensated for by the improvement in PAPR: overall balances with the PAPR reductions taken into account are gains of $0.3 \mathrm{~dB}$ and $1.4 \mathrm{~dB}$ for the roll-off factors of 0.4 and 0.1 , respectively. It should also be noted that the price on decoding complexity to attain these gains is only the doubling of the number of trellis states: it is 8 for the SI with 8-PSK and 4 for the turbo DQPSK.

\section{F. Bit Error Rate in Noncoherent Transmission}

Lastly, iterative decoding of the FBSI in the noncoherent detection has been simulated. The result with the insertion table $\tilde{\tau}_{0.4}$ is shown in Fig. 8 . The error floor observed in the

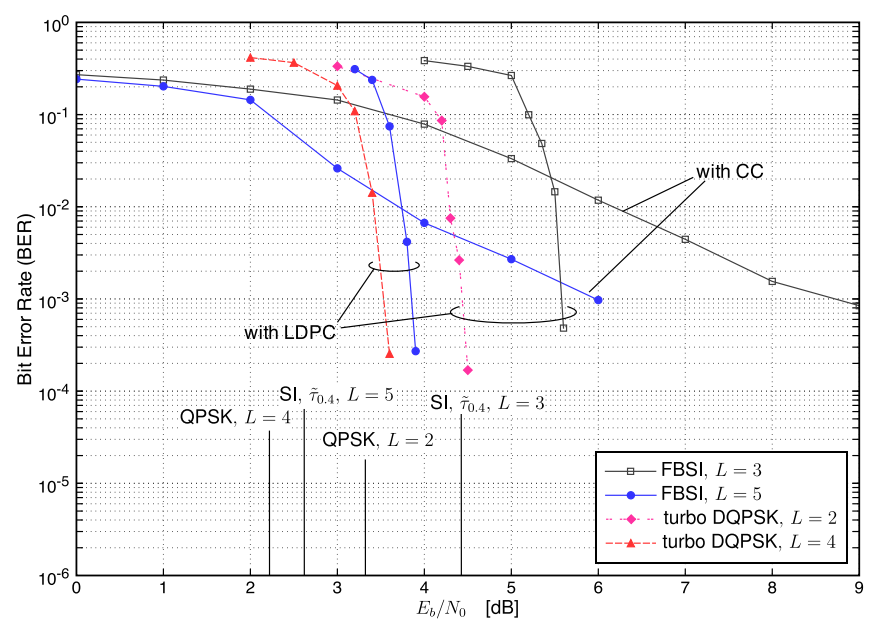

Fig. 8. BER of the FBSI with $\tilde{\boldsymbol{\tau}}_{0.4}$ and the turbo DQPSK in the noncoherent detection. Systems with a comparable complexity are grouped with arcs.

figure may stem from the fact that the recursive nature of the FBSI is canceled by the noncoherence of the receiver. To overcome the error floor, we have also examined the combination of the FBSI with a regular low-density paritycheck (LDPC) code as an outer code, i.e., the CC is now replaced by an LDPC code. The column and row weights of this LDPC code are 3 and 9, respectively, and thus its coding rate is $2 / 3$. We perform 8 iterations between the LDPC and SI decoders, and 10 local iterations within the LDPC decoder at each iteration. The final decision is made by decoding the LDPC code with 50 iterations. The simulation results shown in the same figure exhibit waterfall-like BER curves that are as close as $1.5 \mathrm{~dB}$ from the noncoherent limits.

In the figure, we also plot the performance of the turbo DQPSK with the noncoherent metric derived in [12] and the observed length $L$ chosen such that they have a similar complexity. The employed LDPC code is a rate $-1 / 2$ code having a column weight of 3 and a row weight of 6 , resulting in the same rate $(1 \mathrm{bit} / \mathrm{symbol})$ when combined with QPSK. As observed, the performance gap between the FBSI and turbo DQPSK decreases as the observation length $L$ increases, which can be justified by their AMI characteristics. Note that in this simulation, we have focused on the systems of $1 \mathrm{bit} /$ symbol. However, if a lower rate is targeted, the FBSI can outperform the unconstrained QPSK as suggested by the AMI characteristics (Fig. 6), and thus we can enjoy PAPR reduction with less BER penalty.

\section{CONCLUSION}

In this paper, we have presented a simple modulation code called symbol insertion (SI) for a low-complexity joint design of PAPR reduction and forward error correction in pulse-shaped single-carrier systems. The achievable error rate performance of a serial concatenation employing the SI is 0.6$1.5 \mathrm{~dB}$ worse than those of conventional capacity-approaching concatenated codes with unconstrained constellations. However, the SI can reduce PAPR by $0.9-2.3 \mathrm{~dB}$ and this PAPR reduction is larger than a loss in terms of error rate performance 
for some cases. Furthermore, due to the rotational invariance property, the SI can also be used in the differentially coherent and noncoherent detection scenarios.

\section{APPENDIX}

The conditional entropy $H(\boldsymbol{R} \mid \boldsymbol{S})$ for the noncoherent case can be derived from

$$
H(\boldsymbol{R} \mid \boldsymbol{S})=-\lim _{n \rightarrow \infty} \frac{1}{2 n} E\left[\log _{2} p\left(\boldsymbol{R}_{1}^{n} \mid \boldsymbol{S}_{1}^{n}\right)\right] .
$$

The conditional pdf in the above equation can be expressed as

$$
\begin{aligned}
& p\left(\boldsymbol{R}_{1}^{n} \mid \boldsymbol{S}_{1}^{n}\right) \\
& \quad=\frac{p\left(\boldsymbol{R}_{1}^{n} \mid \boldsymbol{S}_{1}^{n}\right)}{p\left(\boldsymbol{R}_{1}^{n-1} \mid \boldsymbol{S}_{1}^{n-1}\right)} \frac{p\left(\boldsymbol{R}_{1}^{n-1} \mid \boldsymbol{S}_{1}^{n-1}\right)}{p\left(\boldsymbol{R}_{1}^{n-2} \mid \boldsymbol{S}_{1}^{n-2}\right)} \frac{p\left(\boldsymbol{R}_{1}^{n-2} \mid \boldsymbol{S}_{1}^{n-2}\right)}{p\left(\boldsymbol{R}_{1}^{n-3} \mid \boldsymbol{S}_{1}^{n-3}\right)} \cdots .
\end{aligned}
$$

Although each term is conditioned on the sequence of all the past symbols, we observe only $L$ symbols for decoding. Therefore, for arbitrary $k \geq l+1$ we have

$$
\frac{p\left(\boldsymbol{R}_{1}^{k} \mid \boldsymbol{S}_{1}^{k}\right)}{p\left(\boldsymbol{R}_{1}^{k-1} \mid \boldsymbol{S}_{1}^{k-1}\right)}=\frac{\overline{p_{\theta}}\left(\boldsymbol{R}_{k-l+1}^{k}, R_{k-l}^{(i)} \mid \boldsymbol{S}_{k-l+1}^{k}, S^{i_{k-l}}\right)}{\overline{p_{\theta}}\left(\boldsymbol{R}_{k-l+1}^{k-1}, R_{k-l}^{(i)} \mid \boldsymbol{S}_{k-l+1}^{k-1}, S^{i_{k-l}}\right)} .
$$

Moreover, if we assume stationarity, the average of this quantity does not depend on a particular index $k$. Consequently, by choosing $k=l+1$, (42) can be reduced to

$$
H(\boldsymbol{R} \mid \boldsymbol{S})=-\frac{1}{2} E\left[\log _{2} \frac{\overline{p_{\theta}}\left(\boldsymbol{R}_{2}^{l+1}, R_{1}^{(i)} \mid \boldsymbol{S}_{2}^{l+1}, S^{i_{1}}\right)}{\overline{p_{\theta}}\left(\boldsymbol{R}_{2}^{l}, R_{1}^{(i)} \mid \boldsymbol{S}_{2}^{l}, S^{i_{1}}\right)}\right] .
$$

Referring to the definition of $\overline{p_{\theta}}$ in (16), it can be easily confirmed that this quantity does not depend on a particular SI structure. Specifically, for given $L$ independent Gaussian noise samples $W_{1}, \cdots, W_{L}$, we have

$$
\begin{aligned}
& H(\boldsymbol{R} \mid \boldsymbol{S})=H(\boldsymbol{W}) \\
& =\log _{2}\left(\pi N_{0}\right)+\frac{\log _{2} e}{2 N_{0}} E\left[\sum_{n=L-1}^{L}\left(2+2 \Re\left[W_{n}\right]+\left|W_{n}\right|^{2}\right)\right] \\
& \quad+E\left[\log _{2} \frac{I_{0}\left(\frac{2}{N_{0}}\left|L+\sum_{n=1}^{L} W_{n}\right|\right)}{I_{0}\left(\frac{2}{N_{0}}\left|L-2+\sum_{n=1}^{L-2} W_{n}\right|\right)}\right] \\
& =\log _{2}\left(\pi N_{0}\right)+\left(1+\frac{2}{N_{0}}\right) \log _{2} e \\
& +E\left[\log _{2} \frac{I_{0}\left(\frac{2}{N_{0}}\left|L+\sum_{n=1}^{L} W_{n}\right|\right)}{I_{0}\left(\frac{2}{N_{0}}\left|L-2+\sum_{n=1}^{L-2} W_{n}\right|\right)}\right] .
\end{aligned}
$$

The expectation of the third term can be evaluated by a MonteCarlo method. Alternatively, based on the fact that;

$$
\frac{d}{d x} \log _{2} I_{0}(x) \approx \text { const. }, \quad x \gg 1,
$$

we can approximate the third term by

$$
\log _{2} I_{0}\left(\frac{2 L}{N_{0}}\right)-\log _{2} I_{0}\left(\frac{2(L-2)}{N_{0}}\right) .
$$

We have numerically confirmed that this approximation is accurate for $E_{s} / N_{0} \gtrsim 0 \mathrm{~dB}$.

\section{REFERENCES}

[1] J. Wang and J. Chen, "Performance of wideband CDMA systems with complex spreading and imperfect channel estimation," IEEE J. Select. Areas Commun., vol. 19, pp. 152-163, Jan. 2001.

[2] M. Nakamura and H. Torii, "Ternary phase shift keying and its performance," in Proc. IEEE WPMC'02, pp. 1284-1288, Oct. 2002.

[3] M. Chen and O. M. Collins, "Trellis pruning for peak-to-average power ratio reduction," in Proc. IEEE ISIT'05, pp. 1261-1265, Sept. 2005.

[4] S. L. Miller and R. J. O'Dea, "Peak power and bandwidth efficient linear modulation," IEEE Trans. Commun., vol. 46, pp. 1639-1648, Dec. 1998.

[5] M. Tanahashi and H. Ochiai, "Near constant envelope trellis shaping for PSK signaling," IEEE Trans. Commun., vol. 57, pp. 450-458, Feb. 2009.

[6] L. Bahl, J. Cocke, F. Jelinek, and J. Raviv, "Optimum decoding of linear codes for minimizing symbol error rate," IEEE Trans. Inform. Theory, vol. IT-20, pp. 284-287, Mar. 1974.

[7] S. Benedetto, D. Divsalar, G. Montorsi, and F. Pollara, "Serial concatenation of interleaved codes: Performance analysis, design, and iterative decoding," IEEE Trans. Inform. Theory, vol. 44, pp. 909-926, May 1998.

[8] K. R. Narayanan and G. L. Stüber, "A serial concatenation approach to iterative demodulation and decoding," IEEE Trans. Commun., vol. 47, pp. 956-961, July 1999.

[9] P. Hoeher and J. Lodge, "Turbo DPSK: Iterative differential PSK demodulation and channel decoding," IEEE Trans. Commun., vol. 47, pp. 837-843, June 1999.

[10] M. Peleg and S. Shamai (Shitz), "Iterative decoding of coded and interleaved noncoherent multiple symbol detected DPSK," Electron. Lett., vol. 33, pp. 1018-1020, June 1997.

[11] G. Colavolpe, G. Ferrari, and R. Raheli, "Noncoherent iterative (turbo) decoding," IEEE Trans. Commun., vol. 48, Sept. 2000.

[12] I. D. Marsland and P. T. Mathiopoulos, "On the performance of iterative noncoherent detection of coded $M$-PSK signals," IEEE Trans. Commun., vol. 48, pp. 588-596, Apr. 2000.

[13] D. M. Arnold, H. A. Loeliger, P. O. Vontobel, A. Kavčić, and W. Zeng, "Simulation-based computation of information rates for channels with memory," IEEE Trans. Inform. Theory, vol. 52, pp. 3498-3508, Aug. 2006.

[14] D. Divsalar and M. K. Simon, "Multiple-symbol differential detection of MPSK," IEEE Trans. Commun., vol. 38, pp. 300-308, Mar. 1990.

[15] M. Peleg and S. Shamai (Shitz), "On the capacity of the block-wise incoherent MPSK channel," IEEE Trans. Commun., vol. 46, pp. 603609, May 1998.

[16] E. Biglieri, Coding for Wireless Channels. Springer, 2005.

[17] D. Wulich and L. Goldfeld, "Bound of the distribution of instantaneous power in single carrier modulation," IEEE Trans. Wireless Commun., vol. 4, pp. 1773-1778, July 2005.

[18] H. Ochiai, "Power efficiency comparison of OFDM and single-carrier signals," in Proc. IEEE VTC'02 Fall, pp. 899-903, Sept. 2002.

[19] M. Tanahashi and H. Ochiai, "A simple modulation code with peak power reduction and coding gain," in Proc. IEEE ICC'08, pp. 13651369, May 2008.

[20] J. L. Fan, T. L. Poo, and B. H. Marcus, "Constraint gain," IEEE Trans. Inform. Theory, vol. 50, pp. 1989-2001, Sept. 2004.

[21] S. ten Brink, "Convergence behavior of iteratively decoded parallel concatenated codes," IEEE Trans. Commun., vol. 49, pp. 1727-1737, Oct. 2001.

[22] S. Dolinar and D. Divsalar, "Weight distributions for turbo codes using random and nonrandom permutations," JPL-TDA Progress Report 42122, pp. 56-65, Aug. 1995.

PLACE

PHOTO

HERE
Makoto Tanahashi (S'07) was born in Gifu, Japan, in December 1983. He received the B.E., M.E., and $\mathrm{Ph} . \mathrm{D}$. degrees all in electrical engineering from Yokohama National University, Japan, in 2006, 2008, and 2010 respectively. Since April 2009, he has been a Research Fellow of the Japan Society for the Promotion of Science (JSPS).

He was a recipient of a Student Paper Award from the Telecommunications Advancement Foundation in 2009 and the Yasujiro Niwa Outstanding Paper Award in 2010. 
Hideki Ochiai (S'97-M'01) received the B.E. degree in communication engineering from Osaka University, Osaka, Japan, in 1996, and the M.E. and $\mathrm{Ph} . \mathrm{D}$. degrees in information and communication

PLACE PHOTO HERE engineering from the University of Tokyo, Tokyo, Japan, in 1998 and 2001, respectively.

From 1994 to 1995, he was with the Department of Electrical Engineering, University of California, Los Angeles (UCLA), CA, under the scholarship of the Ministry of Education, Science, and Culture. From 2001 to 2003, he was a Research Associate at the Department of Information and Communication Engineering, the University of Electro-Communications, Tokyo, Japan. Since April 2003, he has been with the Department of Electrical and Computer Engineering, Yokohama National University, Yokohama, Japan, where he is currently an Associate Professor. From 2003 to 2004, he was a Visiting Scientist at the Division of Engineering and Applied Sciences, Harvard University, Cambridge, MA.

Dr. Ochiai currently serves as an Editor for IEEE TRANSACTIONS ON WIRELESS COMMUNICATIONS. 